\title{
2 APROXIMACIÓN CONCEPTUAL AL SISTEMA NACIONAL DE INNOVACIÓN
}

Las relaciones entre el cambio tecnológico, la economía y la sociedad es un tema amplio y de mucha especulación e investigación. Algunos investigadores se han concentrado en la visión cultural de Occidente, en las maneras tecnológicas racionales y lineales de pensar. Otros han enfocado sus estudios en la importancia de la tecnología en el sistema capitalista y en la importancia del capitalismo para el cambio tecnológico.

Dentro de esta amplia gama de metodologías para investigar la tecnología y la sociedad, algunos investigadores han tratado de explicar por qué algunos países difieren con respecto al desarrollo tecnológico.

Una cuestión importante que proviene del entendimiento actual entre el desarrollo tecnológico y las naciones es la siguiente: «¿Por qué y como han triunfado algunas naciones en concreto, en producir tanta invención científica e innovación de mercado, que lleva a la difusión y uso de tecnología, mientras que otros países han tenido tantas dificultades?» [McKelvey, 1991, p. 117].

Uno de los enfoques que esta adquiriendo una relevancia especial, actualmente, es el de sistemas nacionales de innovación. Algunos investigadores usan sistemas nacionales de innovación como un término intercambiable de un país a otro, siempre y cuando tengan un sistema similar de $\mathrm{I}+\mathrm{D}$, donde la visión lineal de innovación — desde la ciencia a la tecnología y al crecimiento económico— ha sido frecuentemente usada para justificar las subvenciones gubernamentales de I+D.

Importantes indicadores científicos y tecnológicos han sido desarrollados después de la Segunda Guerra Mundial, pero han habido intentos de medir el «input» de $\mathrm{I}+\mathrm{D}$ - en una empresa, sector o país - y han habido intentos de compararlos con los indicadores económicos de rendimiento que implícitamente aceptan esta progresión lineal básica desde la ciencia al crecimiento económico. En contraste, sin embargo, mucha de la nueva investigación sobre sistemas 
nacionales de innovación ha implicado en un intento de ampliar el alcance analítico, para conseguir una comprensión más profunda de la relación entre el cambio tecnológico y el crecimiento económico.

Sistema nacional de innovación es un término amplio, el cual incluye los procesos de innovación y difusión en el contexto del sistema de producción y en el de las instituciones sociales y económicas. En general el término innovación es comúnmente usado con uno de estos tres significados:

- Para indicar una etapa específica del proceso de cambio tecnológico, cuando un invento es introducido en el mercado ${ }^{1}$.

- Para indicar todo el tipo de novedades no tecnológicas de naturaleza organizacional, social e institucional.

- Para indicar el proceso de creación, difusión o el uso de estos distintos cambios.

Aunque los factores económicos se acentúen, las explicaciones culturales, sociales e históricas han encontrado, en distintos grados, su camino en la investigación teórica en esta área. Esto es porque el proceso de innovación tecnológica puede incluir muchos factores complejos y determinantes, tales como:

- Sectores industriales claves.

- El ambiente nacional.

- Orientaciones culturales.

- Interacciones entre productores - usuarios, etc.

En la descripción de los diferentes enfoques, consideraremos algunos puntos fundamentales que incluyen ${ }^{2}$ :

- La adecuación general de cada teoría en dirigirse a los verdaderos sistemas nacionales de innovación.

- La definición adoptada de tecnología.

- El énfasis relativo en la tecnología o en los procesos económicos de innovación.

- La perspectiva sobre la relación entre el «actor» y la «estructura».

1 Dividiendo el proceso del cambio tecnológico en tres fases - la invención, innovación y difusión - se aclara la diferencia entre hacer una realidad tecnológica desde una idea inicial — la invención—, mercantilizarla — la innovación — y extender esa tecnología a usuarios potenciales o últimos consumidores — la difusión-. Sin embargo, el desarrollo tecnológico es un proceso sin las líneas claras entre las fases porque productor y usuario continúan mejorando el producto [Rosenberg, 1982, en McKelvey, 1991, p. 137].

2 Siguiendo parcialmente a McKelvey [1991, p. 118]. 


\subsection{PROBLEMA COMÚN ¿UN MUNDO NACIONAL O INTERNACIONAL?}

Un problema clave es el que hace referencia al nivel en que los sistemas de innovación han de ser analizados. El asumir la existencia de los sistemas nacionales de innovación implica una integridad significativa y sistémica. ¿Hasta que nivel se integran tales sistemas nacionales importantes, en un mundo cada vez más internacional e integrado, y son las fronteras nacionales la categoría analítica decisiva?

Claramente, esta demarcación es amenazada por la existencia de desproporciones regionales en el desarrollo y crecimiento de la innovación, ambos dentro de países y entre áreas del planeta. La naturaleza desigual del desarrollo capitalista puede hacer más significativo analizar grupos de países — tales como los desarrollados versus los que aún se están desarrollando o el poder de Japón, Europa y EEUU— o, por otra parte, regiones menores — por ejemplo, norte y sur de un país en concreto-.

Además, las presiones internacionales pueden debilitar la autonomía nacional. Por ejemplo, Katzenstein ${ }^{3}$ argumenta que la política industrial flexible es a veces posible en pequeños países desarrollados debido a la existencia de estructuras sociales corporativas, implicando la cooperación del estado, del trabajo y del capital. Sin embargo, la internacionalización económica continúa aumentando, el capital nacional llega a ser internacional y no depende más al mismo nivel del gobierno y de la estructura social nacional.

Un problema con relación al comercio internacional es el de usar naciones como unidad de análisis, ya que el comercio internacional no es simplemente comercio entre países, porque existen las entidades que coordinan las actividades económicas a través de muchos de esos países, empresas específicamente multinacionales. A la vez, las empresas multinacionales ahora tratan de tener identidades múltiples, lo cual significa «intentar llegar a ser compañías locales en muchos países». Las empresas multinacionales forman una parte importante de los sistemas reales de innovación en varios países. Esto tiene implicaciones para cada sistema nacional, mientras al mismo tiempo cada empresa multinacional es un actor relativamente coherente [McKelvey, 1991, p. 119]. Además, muchas políticas nacionales son afectadas y afectan los acontecimientos y a la gente, más allá de las fronteras nacionales, y mucha de la actividad en la ciencia y en la tecnología es claramente internacional.

3 Katzenstein [1985], en McKelvey [1991, p. 119]. 
Sin embargo, el enfoque de los sistemas nacionales de innovación no niega necesariamente que un sistema internacional de innovación pueda existir y exista. Quizá después de identificar las diferencias nacionales —las cuales pueden impedir el error de sobregeneralizar acerca de las características del capitalismola comprensión de cada sistema internacional de innovación puede emerger.

En un enfoque más tradicional de las relaciones internacionales, un sistema internacional de innovación podría ser considerado como la interacción entre países y entre empresas — relativamente— nacionales.

Se puede construir también un mapa de los sistemas internacionales de innovación dentro de un sector industrial en particular, o podría ser de una empresa específica en el sentido de trazar las alianzas estratégicas de la empresa, ubicación de la investigación, interacciones importantes con usuarios, condicionantes de la demanda y cultura de la empresa. El problema es cuánto y donde las fronteras económicas coinciden con las fronteras políticas y nacionales. Estos procesos continuos de internacionalización, obviamente causan problemas para la investigación de la ciencia social, que se diseña para explicar las diferencias nacionales en la capacidad para innovar y difundir la tecnología. A pesar de estos problemas, las naciones todavía constituyen un campo interesante, significativo y útil de análisis, [McKelvey, 1991, p. 119].

Aunque los procesos de internacionalización desafían la soberanía nacional hasta cierto punto, los gobiernos hacen políticas que afectan muchas áreas de la vida corporativa y privada. Además, el complejo sistema de acuerdos políticos nacionales —el bienestar social, el trabajo, las relaciones de mercado- junto con las instituciones y el conjunto aduanero, varía significativamente desde un país a otro. Por estas y otras razones relacionadas, las naciones todavía constituyen una categoría analítica válida.

\subsection{ESTRUCTURA Y ACTOR}

En las ciencias sociales, «estructura» y «actor» frecuentemente se usan para fines opuestos, donde una perspectiva estructural enfatiza los procesos supraindividuales que determinan acciones individuales mientras la perspectiva del actor enfatiza la voluntad libre de los individuos.

Como pregunta Boglind [1989, p. 32], «¿Es la sociedad el resultado cambiante caprichoso de interpretaciones y acciones de individuos, o es un modelo, el cual es creado independientemente del conocimiento y fuerza de voluntad individual del actor?» 
La teoría económica Neoclásica abarca una versión del individualismo metodológico, mientras simultáneamente niega la suficiente diversidad de acción asumiendo preferencias y tecnologías prefijadas, y asumiendo una respuesta racional a cualquier problema de decisión totalmente especificada [Hodgson, 1988, pp. 119—126; Farmer y Matthews, 1991, pp. 103-116].

La teoría de las ventajas comparativas puede ser usada como una ilustración. Bajo ciertas suposiciones argumenta que todos los participantes se benefician si ellos se especializan en la producción y después en el comercio. Esto da un resultado predeterminado. Parece ser que los actores individuales hayan de escoger racionalmente según principios generales, para alcanzar un resultado óptimo y equilibrado. Sin embargo, el estrecho margen de elección que se obtiene a través de la teoría de la ventaja comparativa es ilusoria. Si las suposiciones básicas son más flexibles, y se consideran cambios en el capital y en la tecnología, entonces un número «óptimo» de resultados llega a ser posible. Así los países individualmente pueden actuar para proteger la industria incipiente de las consecuencias negativa del comercio con un país más desarrollado.

Otras líneas tradiciones de investigación tratan de combinar los dos extremos para que estructura y actor se relacionen. Por ejemplo, Edquist [1985] reconoce la diferencia pero intenta usar ambos en su estudio del porqué ciertas técnicas son escogidas en lugar de otras.

El término «social carries of technique», el cual contiene las condiciones necesarias para escoger y actualizar la implantación de una técnica, explícitamente reconoce que «actor es el nivel de libertad de acción determinado por los factores estructurales» [Edquist, 1985, p. 11]. Edquist intenta combinar orden e incertidumbre, limitaciones estructurales y voluntad propia.

Giddens [1984] toma otro enfoque e intenta abarcar, ambos actor y estructura, en su teoría de la estructuración. Giddens argumenta que las estructuras son el medio para y el resultado de la acción social. En otras palabras, cada acción sucede en un ambiente ya estructurado, mientras la acción social a la vez tiene efectos sobre la estructura, o para reproducirla o para cambiarla. La conceptualización de la relación entre el actor y la estructura ensombrece la cuestión de cuánto espacio para la elección o novedad se asume que exista en el proceso de innovación [McKelvey, 1991, p. 121]. 


\subsection{CUATRO ENFOQUES DE SISTEMA NACIONAL DE INNOVACIÓN}

El concepto de sistema nacional de innovación, ahora, tiene un uso amplio. Sin embargo, las definiciones del término varían y es importante analizar las similitudes y las diferencias. En particular, las diferentes definiciones implican cosas diferentes sobre la importancia de las políticas del gobierno y sobre las posibilidades de afectar el futuro. Además, estas definiciones están basadas en diferentes teorías e indican fenómenos empíricos diferentes.

Los investigadores examinados asumen que las diferencias nacionales existen, desde el punto de vista de mercados, instituciones, mecanismos para la introducción y selección de innovaciones, etc., por lo tanto, cada uno de ellos legitiman un nivel de análisis por encima del individuo o de la empresa.

En lugar de la reducción indispensable para explicar todos los fenómenos desde el punto de vista del individuo racional o empresa, estos investigadores implican que los sistemas nacionales tienen su autonomía propia. A distintos niveles, ellos sugieren que el sistema nacional puede representar un nivel de análisis que no es completamente reducible a sus componentes individuales.

De acuerdo con las aspiraciones de explicación universal, con base en las suposiciones generales sobre los mercados y su comportamiento, el enfoque del análisis cambia desde la simple universalidad a la particularidad de las naciones, con la idea que diferencias en el ámbito nacional, — desde el punto de vista de rutinas e instituciones, por ejemplo—, proveerán eventualmente una mejor explicación de la dinámica de los sistemas económicos capitalistas.

Además, las concepciones de dinamismo y del cambio estructural — pueden ser ambas destructiva y creativa como enfatizó Schumpeter [1934 y 1942]— son una parte integral de las teorías aquí presentadas ${ }^{4}$.

Los autores también comparten una idea común de la importancia del cambio tecnológico, aunque ellos difieren con respecto a qué tipo de cambio tecnológico —o innovación- es importante y el por qué. Ellos también difieren con respecto a sí el cambio tecnológico, crecimiento económico y ajuste social son los factores problemáticos en el desarrollo económico.

En la teoría económica ortodoxa, la tecnología es considerada generalmente como conocimiento codificable, lo cuales fácilmente transferible y frecuentemente

4 Si se desea ampliar información sobre Schumpeter, consultar Kamien y Schwartz [1982], que analizan con profundidad las hipótesis Schumpeterianas, el enfoque de la teoría de la decisión y de la teoría de juegos. 
sin coste. En esta tradición ortodoxa, la tecnología es vista como «información que es generalmente aplicable, y fácil de reproducir y usar, donde las empresas pueden producir y usar las innovaciones mediante el acceso libre a un stock o pool general de conocimiento tecnológico» [Dosi, 1988, p. 1130]. En cambio, sin embargo, cuando la tecnología es vista como conocimiento tácito o incodificable [Nelson, 1981, pp. 1029_1064], el desarrollo tecnológico es dependiente de determinadas habilidades históricas y rutinas de investigación. La tecnología por sí misma no puede fácilmente ser transferida a causa de su dependencia de las competencias específicas locales de los individuos.

Teniendo en cuenta estas consideraciones se intentará mostrar que las diferentes definiciones de tecnología son una parte de las diferencias cruciales en la comprensión del dinamismo económico y tecnológico en los sistemas nacionales de innovación.

\subsubsection{ENFOQUE CONCEPTUAL DE PORTER}

En La Ventaja Competitiva de las Naciones, Porter [1990] comparó diez países industrializados 5 . La pregunta detrás de este estudio empírico fue: «نPorqué ciertas empresas están emplazadas en ciertas naciones capaces de innovación consistente? ¿...Porqué ellas persiguen a cualquier coste las mejorías, buscando aún una más sofisticada fuente de ventaja competitiva?» [Porter, 1990, p. 77].

Finalmente, Porter está interesado en el porqué un país específico tiene éxito en industrias específicas. De hecho, el ve un sin sentido comparar naciones a un nivel superior — por ejemplo, actuación global o balanza comercial—. Sin embargo, Porter identifica las características no reducibles de la economía nacional que influye en el éxito de empresas específicas.

Los recursos existentes y la tecnología ya no son la fuente de la ventaja comparativa. Sin embargo, Porter esta a favor de un proceso dinámico, donde las empresas crean la ventaja competitiva a través de la innovación.

La innovación puede ser técnica u organizacional, o ser «una manera nueva de hacer las cosas» y puede ser radical o incremental aunque la innovación en el significado de Porter también incluye un concepto relativamente nuevo — como las radios menores y más económicas—, viendo sus posibilidades, y

5 Los países incluidos en el análisis de Porter son: Dinamarca, Alemania, Italia, Japón, Corea del Sur, Singapur, Suecia, Suiza, UK y EEUU. 
de una forma creadora convirtiéndolo en un producto domésticamente e internacionalmente competitivo.

La base de Porter para comparar los países y las empresas es por lo tanto el cambio económico — particularmente la productividad y el crecimiento-. «El único concepto significativo de competitividad en el ámbito nacional es la productividad. La meta principal de una nación es producir un nivel de vida alto y ascendente para sus ciudadanos» [Porter, 1990, p. 84]. Porter plantea cuatro razones por las cuales las naciones son capaces de apoyar la actividad innovadora en las empresas:

1. Las condiciones de Factor, incluyen no solamente la dotación del recurso existente sino también los factores de producción que la nación puede ayudar a crear. Por ejemplo, las naciones pueden aumentar la reserva de habilidades especializadas necesarias para las empresas y ayudar a desarrollar una base científica. Además, las desventajas nacionales aparentes, tal como una carencia de recursos naturales, pueden estimular políticas privadas o gubernamentales hacia una innovación dinámica.

2. Las condiciones de la Demanda, incluyen la composición y carácter de la demanda nacional. Claramente, las condiciones de la demanda, en sentido cuantitativo y cualitativo, pueden ser un estímulo importante para la innovación si se cumplen ciertas condiciones: si los compradores son sofisticados, si la industria es dominante en el mercado nacional, si los valores relativos al consumo en aquel mercado se expanden internacionalmente. Porter argumenta que la demanda del mercado interno es crucial para la competitividad internacional.

3. El tercer factor en el análisis de Porter son las Industrias Auxiliares y de Soporte, que deben también ser internacionalmente competitivas. Los altos y bajos de las uniones así como las industrias auxiliares proporcionan el acceso a nuevos componentes o a nueva maquinaria, y a los flujos de información, conduciendo al desarrollo de relaciones de funcionamiento íntimas o redes comerciales.

4. Finalmente Porter analiza la Estrategia, Estructura y Rivalidad de las empresas. Porter argumenta que el contexto nacional influye fuertemente en la organización y estrategia de las empresas y el grado de rivalidad entre ellas. La capacidad de las empresas de innovar con éxito en un sector específico depende de este contexto, el sistema nacional de enlaces y relaciones. 
Porter enfatiza que estos cuatro factores deben considerarse conjuntamente como elementos de un todo interactuando entre sí. Solamente uno de los factores no puede explicar el éxito individual de las empresas — examinando las acciones de esta empresa-; cada empresa tiene que ser considerada como parte del sistema nacional. Porter difiere de la teoría económica más tradicional en la inclusión de algunos factores institucionales y contextuales y en su énfasis en la naturaleza dinámica de la competición, donde las empresas representan un papel activo y creativo.

\subsubsection{ENFOQUE CONCEPTUAL DE FREEMAN}

El acercamiento de Christopher Freeman a los sistemas nacionales de innovación dibuja las visiones de sus propios trabajos y de los trabajos conjuntos con Carlota Perez en su camino de investigación sobre la actividad económica [Freeman y Pérez, 1988, pp. 38-66; Pérez, 1985, pp. 441—463].

A su vez, esas teorías son inspiradas por el análisis dinámico de Schumpeter, con su incorporación de cambio tecnológico radical. La teoría de FreemanPerez se basa en el cambio tecnológico, su definición de tecnología se basa en:

- Las innovaciones incrementales. Estas se refieren a cambios pequeños, que son el resultado del proceso de la producción o de los usuarios. Acumulativamente, ellas son una fuente importante de aumentos en la productividad.

- Las innovaciones Radicales. Estas involucran novedad y el desarrollo discontinuo, normalmente el resultado de I+D. Ellas son frecuentemente un trampolín para nuevas inversiones y nuevos mercados.

- Los cambios en el sistema tecnológico. Estos combinan la innovación técnica radical e incremental con la innovación orgánica y administrativa. Ellos frecuentemente involucran un conjunto de tecnologías.

- Los cambios en el paradigma económico-tecnológico. Estos se refieren a los cambios en el meta-paradigma que afecta todos los sectores de la economía, así como también lleva a la creación de nuevos productos y nuevas empresas.

La definición implícita — de Porter- de tecnología, se basa en la información y en los objetos materiales, aunque las categorías [Industrias Auxiliares y de Soporte] y [Estrategia, Estructura y Rivalidad de las empresas] 
también incluyan factores sociales y orgánicos. Cada categoría tiene una implicación diferente para la estructura de los costes y para el dinamismo económico, y la categoría [Estrategia, Estructura y Rivalidad de las empresas] es la base de su teoría económica.

En el enfoque conceptual de Freeman y Perez, la base para la mejoría económica se encuentra en los cambios del paradigma económico—-tecnológico. El cambio en el paradigma se basa en la creación y la difusión de la tecnología radical que contiene nuevo potencial productivo y en la redefinición de eficiencia tecnológica y económica, en todos los sectores de la economía. Sin embargo, «no todos los países tienen que producir las nuevas tecnologías pero tienen que usar el nuevo paradigma económico-tecnológico en su área de especialización» [Freeman y Lundvall, 1988, p. 3]. El cambio tecnológico radical debe, sin embargo, ser acompañado por un nuevo paradigma socio-institucional, que perfeccione el comportamiento social y la política gubernamental, las estructuras institucionales, las maneras de organizar la producción, y así sucesivamente.

La debilitación ocurre cuando el potencial innovador y productivo del «radical viejo»—-la base tecnológica — se pierde. Al final de cada ciclo hay una desigualdad entre las instituciones existentes y la tecnología emergente-dominante y su paradigma. Se explican los crecimientos económicos estructurales a largo plazo y las roturas de las economías capitalista desde el punto de vista de la interacción entre desarrollos tecnológicos radicales y las estructuras institucionales.

Freeman diferencia y analiza los países desde el punto de vista de todo lo bien que ellos han adaptado el nuevo paradigma socio-institucional traído por la creación y la difusión de la tecnología radical. Los sistemas nacionales flexibles tienen más facilidad para ajustar sus paradigmas socio—institucionales a los nuevos requisitos y de esta manera ser vistos como líderes en el nuevo ciclo de tecnología dominante.

Freeman [1987, p. 1] define los sistemas nacionales de innovación como «la red de instituciones en los sectores público y privado cuyas actividades y las interacciones inician, importan, modifican y difunden las nuevas tecnologías». Esta es una definición compleja, pero Freeman se concentra en cuatro aspectos al describir el sistema nacional de innovación Japonés:

1. El papel de la política gubernamental — particularmente del MITI, el Ministerio de Industria y Comercio Internacional-.

2. El papel corporativo de $\mathrm{I}+\mathrm{D}$, especialmente con relación a la tecnología importada.

3. El papel de la educación y entrenamiento. 
4. La estructura general de la industria [Freeman, 1987, p. 4].

Para Freeman, los otros factores socio-institucionales que pueden diferir entre las naciones incluyen:

- La atmósfera de negocio, si es conflictual o consensual.

- La organización del mercado y del sistema de producción.

- El papel del gobierno.

Estos factores representan las instituciones importantes y las maneras de organizar la sociedad, ellos son la clave porque afectan críticamente si una nación será capaz o no de beneficiarse de las nuevas tecnologías.

\subsubsection{ENFOQUE CONCEPTUAL DE LUNDVALL}

Como en el análisis de Porter y Freeman, el enfoque conceptual de BengtAke Lundvall sobre los sistemas nacionales de innovación acentúa la naturaleza dinámica de la actividad económica. En otras palabras, Lundvall, nuevamente sigue la teoría de Schumpeter, analiza el proceso económico como un proceso creador y destructor.

Considerando que Porter solamente pregunta cómo o por qué las empresas en un determinado sector puedan innovar con éxito, Lundvall sube el nivel de abstracción y comparación de las verdaderas diferencias nacionales en la capacidad innovadora y en el resultado económico. Aunque su teoría acentúe la importancia de las empresas y de las estructuras de enlace entre empresas, Lundvall juntamente con Charles Edquist no reduce el nivel de análisis — la nación— en su trabajo empírico.

Como en el enfoque conceptual de Nelson, Lundvall — juntamente con el grupo de Aalborg ${ }^{6}$ - considera el cambio tecnológico como un proceso complejo, dinámico, acumulativo e incierto. Lundvall pone el énfasis en el proceso económico que ayuda la creación y la difusión de la innovación, que incluye pero que no se limita al cambio tecnológico, en lugar del cambio tecnológico, exclusivamente. La innovación puede involucrar cambios en la organización de las empresas, instituciones o mercados, participación de nuevos actores, y así sucesivamente.

En los sistemas capitalistas, Lundvall defiende, que la innovación ocurre dentro de las actividades económicas normales como la producción y comercialización, así como en la $\mathrm{I}+\mathrm{D}$, «en todo momento, en todas las partes

6 El grupo IKE de la Aalborg University es formado por Christopher Freeman de la Science Policy Research Unit, Sussex University, Jan Fagerberg del Institute of Foreign Affairs, Oslo y François Chesnais de la University of Paris — y sus colaboradores - y la OCDE. 
de la economía, nosotros esperamos encontrar procesos continuos de aprendizaje, investigando y descubriendo, produciendo los nuevos productos, las nuevas técnicas, las nuevas formas de organización y los nuevos mercados» [Lundvall 1992, p. 8].

La innovación no es necesariamente una actividad separada efectuada por el departamento de $\mathrm{I}+\mathrm{D}$. La innovación es una parte inherente de las actividades económicas normales y también significa que no es posible identificar el cambio tecnológico como un proceso independiente.

En la teoría de Lundvall, los enlaces entre usuario - comprador- y productor — vendedor - son sumamente importantes para la innovación. A través de la interacción —enlace-, el usuario puede comunicar las necesidades potenciales «demand-pull» y el productor puede comunicar las oportunidades técnicas potenciales «technology-push». Tales interacciones permiten que los actores se agrupen y puedan innovar con más facilidad. En este proceso, Lundvall defiende la importancia de:

- El cambio de información cualitativa.

- Las redes necesarias de comunicación entre actores o empresas.

- Los enlaces entre empresas y sectores en una economía nacional.

En contraste con las ideas neoclásicas, donde normalmente se presume que las metas o las preferencias de las empresas están normalmente fijadas en la determinada e inequívoca información, Lundvall argumenta que estos contactos entre las empresas condicionan sus futuras acciones, porque se conceptualizan los cambios de situación.

Así, la clave para comprender las actividades innovadoras está en las acciones y organización de las empresas y en las interacciones entre ellas: «el aprendizaje interactivo es el centro del proceso de innovación» [Edquist y Lundvall, 1993, p. 267]. Aunque esta breve descripción puede dar la impresión que el nivel microscópico de interacción entre las empresas es el más importante, Lundvall se interesa también en la «estructura determinante de la actividad innovadora» [Freeman y Lundvall, 1988, p. 15].

Cada país se ha interesado en estudiar y fomentar su propio sistema de innovación, basado en las instituciones existentes, recursos históricos, desarrollo industrial y sectores importantes. Edquist y Lundvall [1993, pp. 265-298], comparan los sistemas nacionales de innovación Daneses y Suecos, ellos argumentan que «los límites precisos de sistemas nacionales semejantes deben ser determinados por una combinación del análisis teórico e histórico». En otras palabras, esta teoría especifica dos componentes 
universales importantes de sistemas nacionales de innovación —las instituciones y la estructura industrial — pero no proporciona criterios claros para identificar el más importante.

Según Lundvall, la estructura determinante de innovación en un país —resultando del aprendizaje interactivo- son las instituciones y la estructura económica. Cuanto a las instituciones se refiere a organizaciones, reglas formalizadas y no-formalizadas y normas. Las instituciones se ven como vital en su papel de reguladoras de las actividades económicas, incluyendo la innovación. En las instituciones importantes para el sistema nacional de innovación se incluyen las de regulación del mercado de trabajo, del sistema monetario, normalización de procedimientos, y así sucesivamente. Una estructura institucional especialmente importante es los sistemas formalizados de búsqueda de conocimiento, o actividad de $\mathrm{I}+\mathrm{D}$, dentro de los países, incluyendo las bases de datos nacionales compartidas sobre la tecnología y la economía. La base institucional de estas actividades de investigación es que cada país tiene sus características nacionales específicas y sus propios modelos de comportamiento.

Acerca de la estructura económica, Lundvall se inclina por la tradición estructuralista Francesa. Las interrelaciones hacia delante y hacia atrás «que permiten el learning — by—doing y el learning—by_using» están esencialmente basadas en la micro-formación de enlaces entre las empresas. Pero la completa y compleja estructura industrial que compone un sistema de interacción no puede reducirse a estas relaciones individuales. Dentro de la estructura de producción, Edquist y Lundvall [1993, p. 268] acentúan la importancia de los sectores clave para que el desarrollo económico no se bloquee.

Como Porter [1990] enfatiza que las industrias exitosas en los mercados internacionales son una fuente vital de innovación en la economía nacional. Los bloques de desarrollo son también una fuente importante del dinamismo y de la pujanza económica, especialmente cuando los países pueden tener éxito en crear los nuevos bloques de desarrollo y no simplemente confiar en los productos y sectores industriales tradicionales.

La investigación de Lundvall presenta un intento importante para definir «actor» $\mathrm{y}$ «estructura», y también una discusión profunda de la relación entre la producción y la $\mathrm{I}+\mathrm{D}$. Edquist y Lundvall proporcionan un informe sofisticado de las diferencias nacionales en el sistema de innovación, instituciones y estructuras de la producción, involucrando las interacciones entre las empresas. 


\subsubsection{ENFOQUE CONCEPTUAL DE NELSON}

Nelson y Winter [1982], desarrollan la idea de procesos evolutivos en las economías capitalistas, donde las rutinas en las empresas actúan como «informaciones permanentes». La competición económica conduce a la selección de ciertas rutinas exitosas y seguras, y estas pueden transferirse a otras empresas. En algunos casos la imitación es posible, pero el traslado de las rutinas pueden también ocurrir mediante la compra externa — desde fuera de la empresa—, a través del entrenamiento y movilidad laboral entre otros.

Su teoría evolutiva se aproxima más a la teoría Lamarckiana, donde las características adquiridas pueden pasarse adelante, que del modelo biológico Darviniano donde los genes individuales solamente pueden alterarse mediante la mutación antes del nacimiento y la herencia de características adquiridas se descarta. La teoría Lamarckiana se aplica particularmente en la sociedad porque nuestra evolución cultural se basa en el aprendizaje, considerando que nuestra estructura genética apenas ha cambiado [Gould, 1987, pp. 14-23].

Nelson [1987, p. 7] empieza por identificar las características esenciales del cambio tecnológico en las economías capitalistas. Tres elementos claves de su teoría del cambio tecnológico son:

1. Un mecanismo que «introduce las novedades en el sistema» dando lugar, por eso, a resultados imprevisibles.

2. Algún mecanismo inteligente que «selecciona hacia delante» las entidades presentes en el sistema. Una comprensión de los procesos detrás de la selección de tecnología nos permitiría explicar por qué una solución técnica es escogida y no otra.

3. Los resultados eventuales: «A cualquier tiempo, hay entidades factibles no presentes en el sistema predominante que con una oportunidad de cambio se introducen» [Nelson, 1987, p. 12].

La continua existencia de medios de desarrollo semejantes y factibles significa que el sistema nunca alcanzará un estado de equilibrio final. El proceso permanece dinámico, y nunca alcanzará un punto óptimo. En contraste, la suposición que el sistema puede alcanzar un estado óptimo y perdurable en el tiempo son opciones conocidas.

Según Nelson [1987, p. 12], la diversidad existe porque el cambio tecnológico es un proceso abierto, proceso de multi-caminos, donde ninguna solución mejor a un problema tecnológico puede conocerse o identificarse de antemano. Como una consecuencia, el cambio tecnológico es un proceso muy caro en las economías capitalista, con muchas duplicaciones y «muertes súbitas». 
El proceso de selección y la generación de diversidad son las dos características importantes de este tipo de teoría evolutiva. En ambas teorías, Darviniana y Lamarckiana, el cambio evolutivo, la existencia de variedad, y posiblemente su perfeccionamiento a través de la mutación o recombinación, son cruciales. Nelson identifica el desarrollo pluralista de la tecnología, la continua innovación tecnológica y la competición entre los proyectos, las características claves del sistema capitalista.

La economía evolutiva e institucional difiere de la teoría neoclásica en su definición de tecnología y en su incorporación de cambio tecnológico en el centro de los procesos económicos. Para Nelson, la tecnología se plasma y existe de diferentes formas, como puede ser, la información codificada, el conocimiento público de información, el conocimiento privado poseído -o de artefactos protegidos por patentes- Finalmente existe el conocimiento incodificable o rotinizado, específico de los individuos, de los equipos o de una empresa [Nelson, 1981, pp. 1029-1064].

Nelson argumenta que la mayoría de la tecnología se mueve desde la titularidad privada hasta ser un bien público, que es cuando permite que el conjunto de la sociedad se beneficie. Sin embargo, solamente la titularidad —o propiedad— no identifica si la tecnología generalmente está disponible o no.

Nelson también argumenta que las tecnologías pueden clasificarse según su latente pública. Algunas son más genéricas, teniendo que ver con problemas o reglas abstractas comunes a un campo de la investigación o de la industria mientras otras son más específicas, y están vinculadas a un proceso o producto particular.

Esta definición de tecnología, en otras palabras, significa que la tecnología está incluida en las relaciones sociales de la empresa [Nelson, 1981, pp. 10291064]. El conocimiento implícito de las personas — del trabajador- y la competencia directiva son cruciales para el cambio tecnológico, y especialmente para la empresa en los cambios específicos.

Este es un importante contraste con la representación más mecánica de la empresa en la teoría neoclásica, donde, los gerentes —mando— del proceso de producción toman decisiones racionales con base en los conocimientos codificables.

Esta diferencia es crucial porque Nelson reconoce que aunque los individuos pueden tomar las decisiones y pueden actuar, ellos están limitados por los hábitos conceptuales y las rutinas. Así las instituciones sociales que pueden diferir entre los países pueden amoldar las preferencias y las percepciones y esto puede ser muy importante en el proceso de la innovación. 
Nelson [1988, p. 312] en la investigación del sistema de innovación americano identifica tres características, más bien obvias, de sistemas nacionales de innovación en las economías capitalistas y, que son las siguientes:

1. La gran privatización de la nueva tecnología, que trae la selección de mercado y el móvil de ganancia en la creación de tecnología.

2. La naturaleza evolutiva del cambio tecnológico, particularmente sus múltiples fuentes y su inherentemente proceso de gasto.

3. La confianza depositada en las fuerzas de selección del mercado7.

Nelson argumenta que estas tres características están presentes en las economías capitalistas, a diferencia del antiguo modelo Soviético que era planificado y centralizador.

Además de las empresas u otras partes de la estructura industrial, Nelson enfatiza el papel de las universidades y de los laboratorios de investigación, por lo que se puede ampliar el campo típico del análisis económico. Sin embargo, en su investigación del sistema de innovación americano, Nelson [1988, pp. 312329] se centra en el sector de la I+D. Nelson desarrolló su argumentación como consecuencia de haber llegado a la conclusión de que el sistema de innovación es mayor que el sistema tradicional de la $\mathrm{I}+\mathrm{D}$. Los elementos de estos argumentos incluyen:

- El carácter y eficacia del sistema de innovación de una nación de enseñar, entrenar y volver a entrenar.

- Las relaciones de trabajo como — los modelos de trabajo y dirección, la negociación de gestión, resolución de conflictos-.

- Características de las instituciones financieras.

- Las maneras que se organizan y son controladas las empresas.

Estas instituciones son vitales para producir conocimiento y para difundirlo ampliamente [Nelson y Rosenberg, 1993, p. 13].

En este análisis, las variaciones nacionales en los sistemas capitalista son importantes. Así es, en parte, porque «las naciones difieren en su mix de industrias - sectores industriales - y estas diferencias influyen fuertemente en las formas de los sistemas nacionales de innovación» [Nelson y Rosenberg, 1993, p. 13]. También es porque hay las diferencias entre naciones en la composición y estructura de otras instituciones. Estas instituciones difieren en el papel que

7 Una cuestión polémica sobre la interpretación de Nelson es la siguiente: los agentes no confían simplemente en los resultados del mercado, ellos intentan también anticiparse a través de los condicionantes de los mercados de futuro [McKelvey, 1991, p. 133]. 
juegan, su organización y orientación, cuánto ayudan las empresas nacionales, así como si la financiación es pública o privada.

Las características nacionales del sistema de la $\mathrm{I}+\mathrm{D}$ tiene implicaciones para integrar la ciencia y la tecnología en las actividades de la industria y por lo tanto todo lo fácil o difícil que es para la innovación ocurrir en un país. Los equilibrios diferentes entre la tecnología pública y privada significa que los arreglos institucionales son diferentes para ocultar y para compartir el conocimiento tecnológico y evolucionan a través del tiempo en los diferentes modelos de sistemas nacionales de innovación.

\subsection{OTROS ENFOQUES CONCEPTUALES Y APORTACIONES ACERCA DE SISTEMA NACIONAL DE INNOVACIÓN}

Como ya hablamos anteriormente, sistema nacional de innovación, es un tema amplio, muchos investigadores lo han estudiado. Después de las cuatro aportaciones descritas anteriormente, otros estudios - a nuestro juiciomerecen una distinción pues aportan puntos nuevos a las concepciones de Porter, Freeman, Lundvall y Nelson.

\subsubsection{ENFOQUE CONCEPTUAL DE BELL Y CALLON}

Bell y Callon [1994] a partir del análisis de las relaciones entre los entornos científico y productivo, en el campo de la genética y en varios países, proponen una metodología que permite superar los análisis puramente cualitativos de los sistemas de innovación.

Ellos proponen el concepto de redes técnico-económicas, donde una red es un conjunto coordinado de actores heterogéneos que se relacionan y donde en cualquier etapa del proceso de innovación debe producirse una interacción entre los elementos científicos, tecnológicos y de mercado. Estos elementos deben conocer en todo momento el contexto general en el que se desenvuelven ${ }^{8}$.

8 Las conclusiones que llegan Bell y Callon son las siguientes: Las empresas británicas aprovechan muy poco la avanzada investigación básica de su país, que sirve como fuente de inspiración sobre todo a las empresas japonesas. La investigación académica francesa se ocupa de temas de escaso interés industrial y, cuando se acerca al ámbito tecnológico, es aprovechada fundamentalmente por empresas extranjeras. La investigación académica italiana es un recurso esencial para la tecnología italiana, siendo poco visible para las empresas extranjeras [Bell y Callon, 1994, pp. 67-126]. 
Callon ${ }^{9}$ define la investigación técnico-económica como un conjunto de actores heterogéneos: laboratorios públicos, centros de investigación, organizaciones financieras, empresas, usuarios y proveedores públicos que participan colectivamente en la concepción, elaboración, producción—difusión de los procesos de producción, de bienes y servicios que terminan en una transacción de mercado. La teoría de Bell y Callon se basa en tres elementos claves que son los siguientes:

- La investigación técnico-económica se basa en una infraestructura y está compuesta por características fundamentales, que son: La existencia de reglas escritas o tácitas entre sus miembros, la existencia de condiciones de acceso y la existencia de un mecanismo colectivo de creación de valor resultado de las relaciones estables entre los participantes.

- En cualquier etapa del proceso de innovación debe producirse una interacción entre los elementos científicos, tecnológicos y de mercado que intervienen en la innovación. Estos elementos deben conocer el contexto general en el que se desenvuelven.

- El concepto de investigación técnico-económica está basado esencialmente en la movilidad y en la circulación de la información, independientemente de los soportes que se utilicen y, definiendo un espacio comprendido por todos los actores que crean un círculo que pasa la información necesaria a la realización de su finalidad de manera inteligible.

La figura 2.1 representa estas redes que se articulan en torno a tres polos que $\operatorname{son}^{10}$ :

1. El polo cientifico - C- productor de conocimientos.

2. Elpolo tecnológico - T- que pone a punto las tecnologías — aparatos, especificaciones, capacidades, etc.-, utilizadas en las tareas de producción.

3. El polo de mercado - $\mathrm{M}-$, que representa la demanda existente de bienes y servicios.

9 Callon [1990], en Bell y Callon, 1994, p. 76.

10 Siguiendo a Bell y Callon [1994, pp. 84 -91]. 
FIGURA 2.1. Representación de un sistema nacional de innovación.

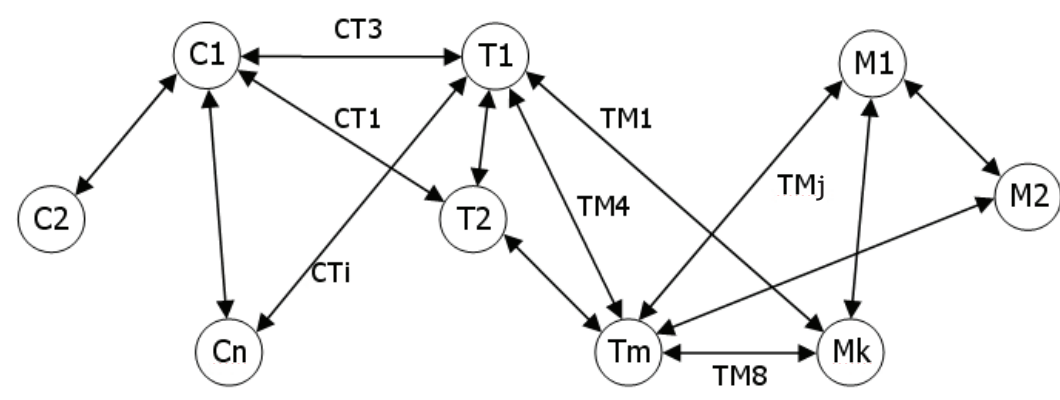

Cn: centro generador de ciencia Tm: centro generador de tecnología Mk: segmento de mercado
Cti: transferencia ciencia-tecnología

Tmj: utilización de la tecnología para un mercado

(C) Bell y Callon [1994, p. 79].

Entres estos polos se sitúan otros dos intermediarios ${ }^{11}$ :

1. El polo CT, que pone en contacto la ciencia y la tecnología.

2. El polo TM que relaciona la tecnología con los mercados y que es formado esencialmente por las empresas.

El análisis de los sistemas de innovación a partir de esta teoría permite la realización de diagnósticos muy precisos. Aparecen sistemas completos, en los que los cinco polos se interrelacionan perfectamente y sistemas discontinuos o dispersos, en los que faltan algunos polos, o bien estos no se relacionan entre si.

En las conclusiones que llegaran Bell y Callon en su trabajo en el campo de la genética, queda claro que es frecuente encontrar polos científicos aislados, sin ningún contacto con las necesidades de las empresas y de los mercados.

El conocimiento de los sistemas de innovación es de una gran utilidad para el diseño y desarrollo de las correspondientes políticas científicas, tecnológicas y de mercado [Escorsa, 1996b, p. 5].

\subsubsection{ENFOQUE CONCEPTUAL DE FERNÁNDEZ Y CONESA}

A través del proyecto financiado por la Dirección General XIII, Dirección D, de la Comisión Europea - CCE_- de un estudio complementario financiado

11 Siguiendo a Bell y Callon [1994, pp. 88-90]. 
por la Comisión Interministerial de Ciencia y Tecnología de España - CICYTy de la publicación de la investigación por la Comunidad Valenciana, Fernández y Conesa [1996], hacen un análisis del Sistema Español de Innovación —SEI—y de los Sistemas Regionales de Innovación —SRI— que lo integran.

El enfoque utilizado en la investigación fue el enfoque interactivo del proceso de innovación. El enfoque interactivo permite el estudio de los procesos de innovación a través del análisis de sistemas. Asimismo, y de acuerdo con las recomendaciones de la OCDE [1992], Fernández y Conesa consideraron que la innovación y su difusión son dos aspectos de un mismo proceso y no dos actividades distintas.

En este contexto, «se define sistema nacional de innovación como el conjunto de elementos y estructuras, que asumen funciones especificas en el proceso de producción, transmisión y almacenamiento de conocimientos y por su capacidad de interacción, mediante la articulación de sus elementos, para la producción de conocimientos y la difusión y utilización de los mismos» [Fernández y Conesa, 1996, p. 48].

Fernández y Conesa consideraran, además, que los elementos del SNI se agrupan, de acuerdo con su actividad principal dentro del proceso de innovación, en una serie de entornos ${ }^{12}$ :

- El entorno científico, en el que mayoritariamente se producen conocimientos científicos.

- El entorno tecnológico, en el que se desarrollan las tecnologías.

- El entorno productivo, que produce bienes y servicios, aportando un valor añadido.

- El entorno financiero, que ofrece recursos financieros a los demás entornos para el desarrollo de sus respectivas actividades.

La adquisición por parte de las empresas de conocimientos científicos y técnicos no es automática ni los cauces para lograrlo están establecidos ni es fácil su consecución. Para que las empresas puedan aprovechar dichos conocimientos necesitan que esos se encuentren disponibles de forma adecuada y tener la

12 Además de los que se citan, se podría incluir también el «entorno de los usuarios», formado por las personas que finalmente demandan productos o servicios que incorporan tecnología. En España, este entorno apenas contiene elementos activos, es decir, aún no se ha organizado suficientemente [a través de asociaciones, sindicatos, ayuntamientos, etc.] para implicarse en los procesos de innovación tecnológica; de ahí que para una mayor simplicidad no se haya incluido en el enfoque de la investigación del Sistema Español de Innovación, [Fernández y Conesa, 1996, p. 27]. 
capacidad para gestionarlos, es decir, poseer una organización y unos recursos humanos con la formación y experiencia necesarias para integrarlos en sus políticas de desarrollo [Fernández y Conesa, 1996, p. 44].

Si este proceso no se da, los resultados de la I+D obtenidos por los científicos pueden no emplearse para resolver los problemas tecnológicos que se plantean en los diferentes sectores de la actividad.

La capacidad de interacción de un SNI se considera particularmente importante porque, si no se producen relaciones entre sus diversos elementos no se podrá hablar realmente de un «Sistema de Innovación» sino, en el mejor de los casos, de un conjunto de elementos más o menos activos o innovadores.

En este sentido, en los países en cuyos tejidos empresariales predominan las pequeñas y medianas empresas [PYME] se ha podido comprobar que las relaciones de este tipo de empresas con los otros elementos del Sistema - especialmente con los centros públicos de investigación y con las agencias de financiación de la innovación — no son fáciles ni automáticas por muchas y diversas razones —escasez de personal técnico, falta de tiempo para las relaciones, dificultades de comunicación, diversidad de objetivos y preocupaciones, etc.- - Por consiguiente, si las relaciones son necesarias pero no se producen espontáneamente — al menos en la cuantía y calidad precisasse han de promover mediante instrumentos de política científica y tecnológica y se han de propiciar mediante entidades capaces de lograr una comunicación fluida entre los diversos elementos del sistema nacional de innovación [Fernández y Conesa, 1996, p. 28]. 
FIGURA 2.2. Representación del sistema español de innovación.

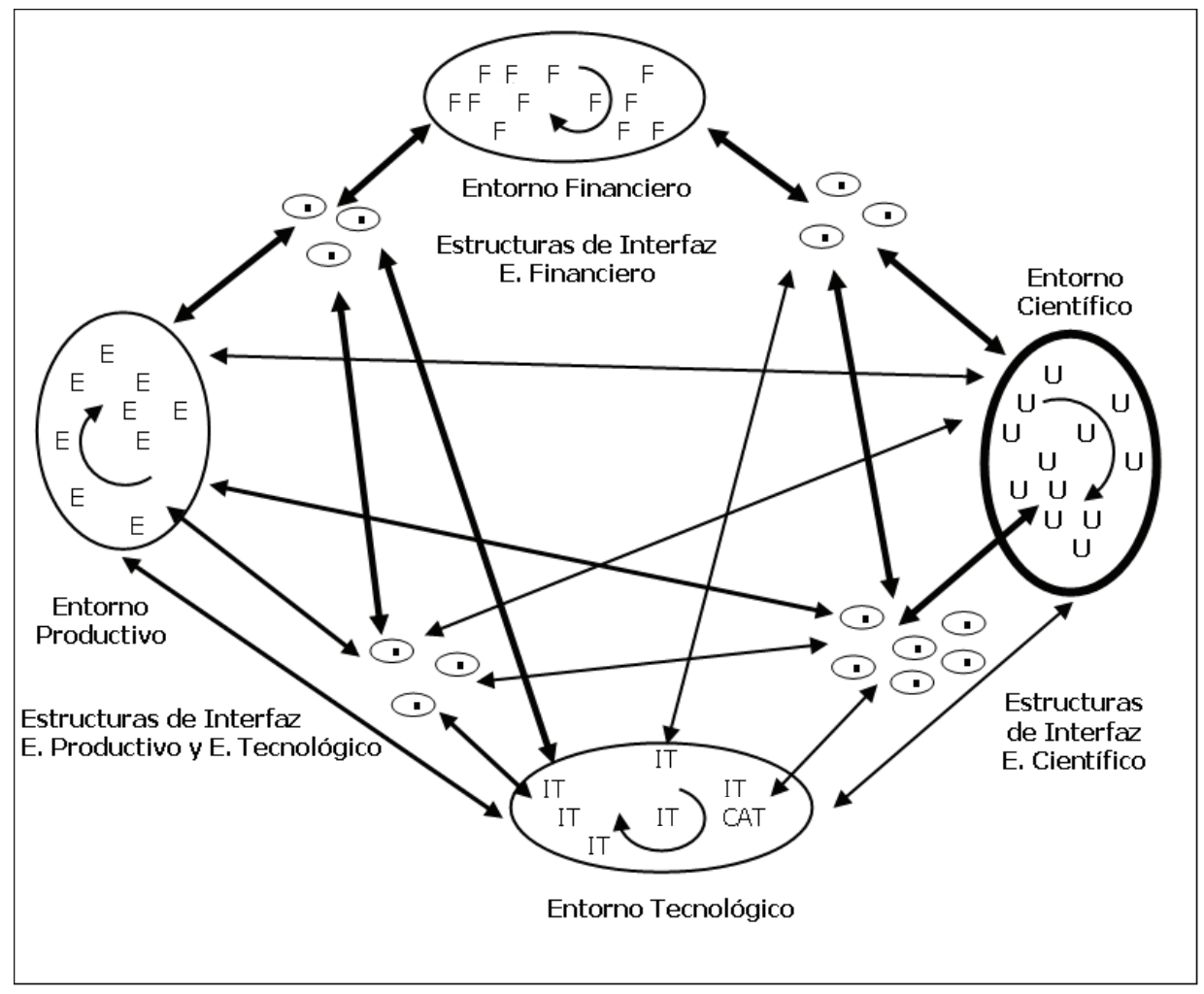

(C) Fernández y Conesa [1996, p. 53].

\subsection{CONCLUSIONES DEL CAPÍTULO}

En el presente capítulo se ha pretendido hacer un resumen de las teorías explicativas del Sistema Nacional de Innovación. Este resumen necesariamente breve, tuvo como finalidad la descripción de un marco teórico de referencia que permite la ubicación de las particularidades a estudiar. La abundante literatura existente sobre el tema nos ha obligado a ser selectivos a la hora de escoger aquellas teorías o enfoques que fundamentan nuestro trabajo. La no-inclusión de determinadas teorías o enfoques no presupone un menosprecio de éstos o aquellas. Al contrario pensamos que en general, cada uno de los diferentes puntos de vista aporta algún valor que ayuda a la compresión del fenómeno Sistema de Innovación.

En el análisis de las principales abordajes conceptuales de SNI, los cuatro autores Porter, Freeman, Lundvall y Nelson tienen una concepción diferente que se resumen de la siguiente manera: En la visión de Porter no es posible analizar 
las diferencias nacionales, analizando únicamente un sector industrial o industrias específicas con éxitos en un país. El sistema de innovación se refiere al ambiente que apoya la actividad innovadora de la empresas. El foco empírico del análisis de Porter es la comparación entre industrias. Para Freeman una nueva y radical tecnología promueve la innovación social e institucional a nivel nacional. Por otra parte la innovación tecnológica en su concepción puede ser incremental. El foco empírico del análisis de Freeman es la comparación entre naciones con base en sus innovaciones y ajustes en sus instituciones sociales. En el enfoque de Lundvall el sistema de innovación se refiere a la economía nacional. Lundvall señala la importancia de los enlaces y las interacciones dentro de los bloques de desarrollo. El sistema de nacional de innovación es formado por las instituciones pertinentes y la estructura industrial. El foco empírico del análisis de Lundvall es el desarrollo histórico de las instituciones y la estructura productiva de los diferentes países. También para Nelson el sistema de innovación se refiere a la economía nacional. Las diferencias en la estructura industrial —necesidades de la industria en ciencia y tecnología y si la tecnología es pública o privada- y en la organización de las instituciones — especialmente en el sistema de I+D — explican como los sistemas nacionales de innovación difieren. El enfoque empírico del análisis de Nelson es la diferencia institucional actual entre las naciones.

Después de haber hecho un repaso a los diferentes enfoques y teorías y aunque sus definiciones de tecnología difieren, los autores acentúan el papel dinámico que ejerce la innovación en la economía. La pregunta que surge después de este análisis es la siguiente: ¿Qué es un sistema de innovación? El sistema de innovación significa algo más que los aspectos cuantificables de los sistemas de $\mathrm{I}+\mathrm{D}$ nacionales. El sistema de $\mathrm{I}+\mathrm{D}$, corporativo o financiado por el gobierno, es una pieza importante en la capacidad que tienen las naciones para innovar y difundir la tecnología, pero no suficiente para describir sus diferencias. El concepto de sistema de innovación es una herramienta analítica útil para comprender esas diferencias en el tipo y proporción de las innovaciones. El cambio tecnológico depende principalmente de la sinergia que pueda haber, en el sistema de innovación, entre la potencialidad tecnológica, oportunidades del mercado, instituciones y de la estructura de la economía, que son todos afectados por la estructura específica de relaciones sociales en un área territorial. 
